\title{
498.
}

\section{ON THE INVERSION OF A QUADRIC SURFACE.}

[From the Quarterly Journal of Pure and Applied Mathematics, vol. XI. (1871), pp. 283-288.]

THE inversion intended to be considered is that by reciprocal radius vectors, viz. if $x, y, z$ are rectangular coordinates, and $r^{2}=x^{2}+y^{2}+z^{2}$, then $x, y, z$ are to be changed into $\frac{x}{r^{2}}, \frac{y}{r^{2}}, \frac{z}{r^{2}}$. But it is convenient to introduce for homogeneity a fourth coordinate $w,=1$; and the change then is $x, y, z$ into $\frac{x w^{2}}{r^{2}}, \frac{y w^{2}}{r^{2}}, \frac{z w^{2}}{r^{2}}$.

Starting from the quadric surface

$$
\left(a, b, c, d, f, g, h, l, m, n \gamma(x, y, z, w)^{2}=0,\right.
$$

or, what is the same thing,

$$
\begin{aligned}
& \left(a, b, c, f, g, h \gamma(x, y, z)^{2}\right. \\
+ & 2 w(l x+m y+n z) \\
+ & d w^{2}
\end{aligned}
$$

the equation of the inverse surface is

$$
\begin{aligned}
& w^{2}(a, b, c, f, g, h \gamma x, y, z)^{2} \\
+ & 2 w(l x+m y+n z) r^{2} \\
+ & d r^{4}=0
\end{aligned}
$$

where $r^{2}=x^{2}+y^{2}+z^{2}$. The inverse surface is thus a quartic having the nodal conic $w=0, x^{2}+y^{2}+z^{2}=0$ (circle at infinity); and having the node $x=0, y=0, z=0$ (the centre of inversion); or say it is a nodal bicircular quartic surface, or nodal anallagmatic. 
For $x, y, z$ write $x-\frac{1}{2} \frac{l}{d} w, y-\frac{1}{2} \frac{m}{d} w, z-\frac{1}{2} \frac{n}{d} w$, and put for shortness

$$
\begin{aligned}
& l x+m y+n z=u, l^{2}+m^{2}+n^{2}=\alpha, \\
& a l+h m+g n=\mathrm{a}, \quad(a, b, c, f, g, h \gamma l, m, n)^{2}=A, \\
& h l+b m+f n=\mathrm{b}, \\
& g l+f m+c n=\mathrm{c},
\end{aligned}
$$

then

$$
\begin{array}{ccc}
r^{2} & \text { becomes } & r^{2}-\frac{u w}{d}+\frac{1}{4} \frac{\alpha}{d^{2}} w^{2}, \\
l x+m y+n z \quad & \quad u-\frac{1}{2} \frac{\alpha}{d} w \\
(a, \ldots \gamma x, y, z)^{2} & \quad & (a, \ldots \gamma x, y, z)^{2}-(\mathrm{a} x+\mathrm{b} y+\mathrm{c} z) \frac{w}{d}+\frac{1}{4} A \frac{w^{2}}{d^{2}} .
\end{array}
$$

Hence the equation is

$$
\begin{aligned}
& d\left\{r^{4}-2 r^{2} \frac{u w}{d}+w^{2}\left(\frac{1}{2} \frac{\alpha}{d^{2}} r^{2}+\frac{u^{2}}{d^{2}}\right)-\frac{1}{2} \frac{\alpha u w^{2}}{d^{3}}+\frac{1}{16} \frac{\alpha^{2}}{d^{4}} w^{4}\right\} \\
+ & 2\left(w r^{2}-\frac{u w^{2}}{d}+\frac{1}{4} \frac{\alpha}{d^{2}} w^{3}\right)\left(u-\frac{1}{2} \frac{\alpha}{d} w\right) \\
+ & w^{2}\left\{(a, \ldots \gamma x, y, z)^{2}-(\mathrm{a} x+\mathrm{b} y+\mathrm{c} z) \frac{w}{d}+\frac{1}{4} A \frac{w^{2}}{d^{2}}\right\}=0
\end{aligned}
$$

viz. arranging and reducing, this is

$$
\begin{aligned}
& d r^{4} \\
& +w^{2}\left\{-\frac{1}{2} \frac{\alpha}{d} r^{2}-\frac{u^{2}}{d}+(a, \ldots \gamma x, y, z)^{2}\right\} \\
& +w^{3}\left\{\frac{\alpha u}{d^{2}}-\frac{1}{d}(\mathrm{a} x+\mathrm{b} y+\mathrm{c} z)\right\} \\
& +w^{4}\left\{-\frac{3}{16} \frac{\alpha^{2}}{d^{3}}+\frac{1}{4} A \frac{1}{d^{2}}\right\}=0 ;
\end{aligned}
$$

and we may without loss of generality assume

$$
\begin{array}{ll}
-\frac{m n}{d}+f=0, \text { that is } & d f-m n=0, \\
-\frac{n l}{d}+g=0, \quad, & d g-n l=0, \\
-\frac{l m}{d}+h=0, \quad, & d h-l m=0 .
\end{array}
$$


The equation then is

$$
\begin{aligned}
& +w^{2}\left\{-\frac{1}{2} \frac{\alpha}{d^{2}}\left(x^{2}+y^{2}+z^{2}\right)+\left(\frac{a}{d}-\frac{l^{2}}{d^{2}}\right) x^{2}+\left(\frac{b}{d}-\frac{m^{2}}{d^{2}}\right) y^{2}+\left(\frac{c}{d}-\frac{n^{2}}{d^{2}}\right) z^{2}\right\} \\
& +w^{3}\left\{\frac{\alpha u}{d^{3}}-\frac{1}{d^{2}}(a x+b y+c z)\right\} . \\
& +w^{4}\left\{-\frac{3}{16} \frac{\alpha^{2}}{d^{4}}+\frac{1}{4} A \frac{1}{d^{3}}\right\}=0 .
\end{aligned}
$$

Write

We have

$$
\begin{aligned}
& a d-l^{2}=a^{\prime} d \\
& b d-m^{2}=b^{\prime} d \\
& c d-n^{2}=c^{\prime} d
\end{aligned}
$$

$$
a=a l+h m+g n=g n=a l+\frac{l m^{2}}{d}+\frac{l n^{2}}{d}=\frac{l}{d}\left(a d-l^{2}+\alpha\right)
$$

that is

$$
a=l a^{\prime}+\frac{l \alpha}{d}
$$

and similarly

$$
\begin{aligned}
& \mathrm{b}=m b^{\prime}+\frac{m \alpha}{d}, \\
& \mathrm{c}=n c^{\prime}+\frac{n \alpha}{d} .
\end{aligned}
$$

Hence also

$$
A=l^{2} a^{\prime}+m^{2} b^{\prime}+n^{2} c^{\prime}+\frac{a^{2}}{d}
$$

and the equation is

$$
\begin{aligned}
& +w^{2}\left\{\left(-\frac{1}{2} \frac{\alpha}{d^{2}}+\frac{a^{\prime}}{d}\right) x^{2}+\left(-\frac{1}{2} \frac{\alpha}{d^{2}}+\frac{b^{\prime}}{d}\right) y^{2}+\left(-\frac{1}{2} \frac{\alpha}{d^{2}}+\frac{c^{\prime}}{d}\right) z^{2}\right\} \\
& +w^{3}\left\{-\frac{l a^{\prime}}{d^{2}} x-\frac{m b^{\prime}}{d^{2}} y-\frac{n c^{\prime}}{d^{2}} z\right\} \\
& +w^{4}\left\{\frac{1}{4 d^{3}}\left(l^{2} a^{\prime}+m^{2} b^{\prime}+n^{2} c^{\prime}\right)+\frac{1}{16} \frac{\alpha^{2}}{d^{4}}\right\}=0 .
\end{aligned}
$$

This is Kummer's form, say

$$
r^{4}=4 w^{2}\left\{\alpha_{1} x^{2}+\beta_{1} y^{2}+\gamma_{1} z^{2}+\delta_{1} w^{2}+2 w\left(a_{1} x+b_{1} y+c_{1} z\right)\right\},
$$


where

$$
\begin{aligned}
& -4 \alpha_{1}=-\frac{1}{2} \frac{\alpha}{d^{2}}+\frac{a^{\prime}}{d} \\
& -4 \beta_{1}=-\frac{1}{2} \frac{\alpha}{d^{2}}+\frac{b^{\prime}}{d} \\
& -4 \gamma_{1}=-\frac{1}{2} \frac{\alpha}{d^{2}}+\frac{c^{\prime}}{d} \\
& -4 \delta_{1}=\frac{1}{4 d^{3}}\left(l^{2} a^{\prime}+m^{2} b^{\prime}+n^{2} c^{\prime}\right)+\frac{1}{16} \frac{\alpha^{2}}{d^{4}} \\
& -8 a_{1}=-\frac{l a^{\prime}}{d^{2}} \\
& -8 b_{1}=-\frac{m b^{\prime}}{d^{2}} \\
& -8 c_{1}=-\frac{n c^{\prime}}{d^{2}}
\end{aligned}
$$

Hence Kummer's equation

$$
\delta_{1}+\lambda^{2}=\frac{a_{1}^{2}}{\lambda+\alpha_{1}}+\frac{b_{1}^{2}}{\lambda+\beta_{1}}+\frac{c_{1}^{2}}{\lambda+\gamma_{1}}
$$

or say

$$
64 \delta_{1}+64 \lambda^{2}=\frac{256 a_{1}^{2}}{4 \lambda+4 x_{1}}+\frac{256 b_{1}^{2}}{4 \lambda+4 \beta_{1}}+\frac{256 c_{1}^{2}}{4 \lambda+4 \gamma_{1}}
$$

becomes

$$
64 \lambda^{2}-\frac{4}{d^{3}}\left(l^{2} a^{\prime}+m^{2} b^{\prime}+n^{2} c^{\prime}\right)-\frac{\alpha^{2}}{d^{4}}=\frac{4 l^{2} a^{\prime 2}}{d^{4}\left(\frac{1}{2} \frac{\alpha}{d^{2}}-\frac{a^{\prime}}{d}+4 \lambda\right)}+\frac{4 m^{2} b^{\prime 2}}{d^{4}\left(\frac{1}{2} \frac{\alpha}{d^{2}}-\frac{b^{\prime}}{d}+4 \lambda\right)}+\frac{4 n^{2} c^{\prime 2}}{d^{4}\left(\frac{1}{2} \frac{\alpha}{d^{2}}-\frac{c^{\prime}}{d}+4 \lambda\right)},
$$

which is satisfied by $4 \lambda=-\frac{1}{2} \frac{\alpha}{d^{2}}$. Writing therefore

$$
1 \lambda+\frac{1}{2} \frac{\alpha}{d^{2}}=-\frac{\theta}{d}
$$

that is

$$
\begin{gathered}
8 \lambda=-\frac{2 \theta}{d}-\frac{\alpha}{d^{2}} \\
64 \lambda^{2}=\frac{4 \theta^{2}}{d^{2}}+\frac{4 \theta \alpha}{d^{3}}+\frac{\alpha^{2}}{d^{4}}
\end{gathered}
$$

the equation is

$$
\frac{4 \theta^{2}}{d^{3}}+\frac{4 \theta \alpha}{d^{3}}-\frac{4}{d^{3}}\left(l^{2} a^{\prime}+m^{2} b^{\prime}+n^{2} c^{\prime}\right)=\frac{4 l^{2} a^{\prime 2}}{d^{4}\left(-\frac{\theta}{d}-\frac{a^{\prime}}{d}\right)}+\frac{4 m^{2} b^{\prime 2}}{d^{4}\left(-\frac{\theta}{d}-\frac{b^{\prime}}{d}\right)}+\frac{4 n^{2} c^{\prime 2}}{d^{4}\left(-\frac{\theta}{d}-\frac{c^{\prime}}{d}\right)},
$$

viz. this is

$$
l^{2} a^{\prime}+m^{2} b^{\prime}+n^{2} c^{\prime}-\theta \alpha-\theta^{2} d=\frac{l^{2} a^{\prime 2}}{\theta+a^{\prime}}+\frac{m^{2} b^{\prime 2}}{\theta+b^{\prime}}+\frac{n^{2} c^{\prime 2}}{\theta+c^{\prime}}
$$


which is of course satisfied by $\theta=0$. Moreover the derived equation

$$
-\alpha-2 \theta d=-\frac{l^{2} a^{\prime 2}}{\left(\theta+a^{\prime}\right)^{2}}-\frac{m^{2} b^{\prime 2}}{\left(\theta+b^{\prime}\right)^{2}}-\frac{n^{2} c^{\prime 2}}{\left(\theta+c^{\prime}\right)^{2}}
$$

is also satisfied by $\theta=0$, so that this is a double root. The equation in fact is

$$
\begin{aligned}
\left\{\theta^{2} d+\theta \alpha-\right. & \left.\left(l^{2} a^{\prime}+m^{2} b^{\prime}+n^{2} c^{\prime}\right)\right\}\left(\theta+a^{\prime}\right)\left(\theta+b^{\prime}\right)\left(\theta+c^{\prime}\right) \\
+ & \left\{l^{2} a^{\prime 2}\left(\theta+b^{\prime}\right)\left(\theta+c^{\prime}\right)+m^{2} b^{\prime 2}\left(\theta+c^{\prime}\right)\left(\theta+a^{\prime}\right)+n^{2} c^{\prime 2}\left(\theta+a^{\prime}\right)\left(\theta+b^{\prime}\right)\right\}=0,
\end{aligned}
$$

or, expanding and dividing by $\theta^{2}$, this is

$$
\begin{aligned}
& d\left(\theta+a^{\prime}\right)\left(\theta+b^{\prime}\right)\left(\theta+c^{\prime}\right) \\
+ & \alpha\left\{\theta^{2}+\theta\left(a^{\prime}+b^{\prime}+c^{\prime}\right)+b^{\prime} c^{\prime}+c^{\prime} a^{\prime}+a^{\prime} b^{\prime}\right\} \\
- & \left(l^{2} a^{\prime}+m^{2} b^{\prime}+n^{2} c^{\prime}\right)\left(\theta+a^{\prime}+b^{\prime}+c^{\prime}\right) \\
+ & l^{2} a^{\prime 2}+m^{2} b^{\prime 2}+n^{2} c^{\prime 2}=0,
\end{aligned}
$$

which gives the remaining three roots.

If $a^{\prime}=b^{\prime}=c^{\prime}$ the equation is

$$
\left(\theta+a^{\prime}+\alpha\right)\left(\theta+a^{\prime}\right)^{2}=0 .
$$

I recall that we have

$$
\begin{aligned}
& a, b, c, d, \quad f=\frac{m n}{d}, \quad g=\frac{n l}{d}, \quad h=-\frac{l m}{d}, \quad l, m, n, \\
& a^{\prime}=a-\frac{l^{2}}{d}, \quad b^{\prime}=b-\frac{m^{2}}{d}, \quad c^{\prime}=c-\frac{n^{2}}{d}, \quad \alpha=l^{2}+m^{2}+n^{2},
\end{aligned}
$$

so that the quadric surface is

$$
d\left(a^{\prime} x^{2}+b^{\prime} y^{2}+c^{\prime} z^{2}\right)+(l x+m y+n z+d w)^{2}=0,
$$

and that, $\alpha_{1}, \beta_{1}, \gamma_{1}, \delta_{1}, a_{1}, b_{1}, c_{1}$ denoting as before, the equation of the inverse surface (referred to a different origin) is

$$
r^{4}=4 w^{2}\left\{\alpha_{1} x^{2}+\beta_{1} y^{2}+\gamma_{1} z^{2}+\delta_{1} w^{2}+2 w\left(a_{1} x+b_{1} y+c_{1} z\right)\right\} .
$$

